\title{
September 2013 Banner Good Samaritan I Phoenix VA Critical Care Journal Club
}

\author{
Raschke RA, Curry SC, Warkentin TE, Gerkin RD. improving clinical interpretation \\ of the anti-platelet factor 4/heparin enzyme-linked immunosorbent assay for the \\ diagnosis of heparin-induced thrombocytopenia through the use of receiver \\ operating characteristic analysis, stratum-specific likelihood ratios, and Bayes \\ theorem. Chest. 2013;144(4):1269-75. [CrossRef] [PubMed]
}

I have to apologize for being a little self-serving in choosing my own publication for review, but I wanted to crow a little, and relate a small personal experience regarding bedside research. To this end, I'm going to discuss how the research came about rather than offering critical appraisal. Nick Sparacino did a great job reviewing this article in conference without stepping on the toes of his program director (sorry to put you in that difficult position Nick!).

Our study reanalyzed previously published data on 1958 patients with suspected heparin-induced thrombocytopenia, and showed that the current cutoff for a positive anti-heparin/PF4 ELISA (HIT ELISA) of 0.4 OD is suboptimal - leading to the confusing situation in which a mildly "positive" test result in the 0.4-1.0 range actually rules HIT out. Receiver operating curve analysis shows that the optimal cutoff for the discriminant accuracy of the HIT ELISA is $0.8 \mathrm{OD}$, and that interpretation can be further improved by using an algorithm that utilizes clinical suspicion and a stratified interpretation of the test result. This algorithm allows confident diagnostic and treatment decisions to be made in about $90 \%$ without the need for confirmation by serotonin release assay (SRA).

The idea for this publication came from one of our fellowship M\&M conferences, in which we presented a patient with an acute right-sided myocardial infarction caused by HIT. A discussion ensued regarding the sensitivity and specificity of the HIT ELISA, and we were left confused by the section on HIT diagnosis in UptoDate ${ }^{\circledR}$, which stated that a mildly positive ELISA result essentially ruled HIT out. I pulled the reference (for which Dr. Warkentin was the lead author), and began re-analyzing the tabulated data in the publication with help from Dr. Curry, Dr. Gerkin and Dr. Jack Peirce. We called the manufacturer of the HIT ELISA and talked to several company representatives to learn that the manufacturer cut off for diagnostic interpretation of the ELISA result was determined using the distribution of results in normal healthy volunteers, not with data on patients suspected of having HIT. This seemed highly suspect. We contacted Dr. Warkentin (in my opinion the world's leading expert on HIT) and he graciously agreed to supply his original data and work with us to reanalyze it. It was an honor to work with Dr. Warkentin. I think it's safe to say that we all learned an astonishing amount about ROC curves, which turn out to be far more interesting than any of us originally imagined. This interest has led to several didactic sessions on ROC curves in our fellowship conferences, and for the Mayo Pulmonary fellowship. Our fellow Tonya Whiting is currently working on a validation study for the HIT ELISA algorithm, which is in process to implement throughout Banner Health. It is fascinating to me that a simple clinical question that arose out of one of our M\&M conferences led to such a cascade of benefits. This is a good example of benefits fellowship training brings to our institutions. 
Hung IF, To KK, Lee CK, Lee KL, Yan WW, Chan K, Chan WM, Ngai CW, Law KI, Chow FL, Liu R, Lai KY, Lau CC, Liu SH, Chan KH, Lin CK, Yuen KY. Hyperimmune IV immunoglobulin treatment: a multicenter double-blind randomized controlled trial for patients with severe 2009 influenza $A(H 1 N 1)$ infection. Chest. 2013;144(2):464-73. [CrossRef] [PubMed]

This study was a small randomized controlled trial with a primary outcome of mortality. However, the methods of this study are not adequate to support the author's conclusion that hyperimmune IV immunoglobulin $(\mathrm{H}-\mathrm{IVIg})$ benefits mortality of patients with severe H1N1 influenza if given within five days. The primary outcome analysis of 34 patients presented in table 1 shows that five of seventeen patients who received $\mathrm{H}$-IVIg died compared to four of seventeen control patients (obviously no benefit there). The authors subsequently performed a subgroup analysis of 22 patients who received treatment within five days of symptom onset. All five of the $\mathrm{H}$-IVIg fatalities are excluded from this analysis, but all four of the control deaths are retained. This is not explicitly stated by the authors, but can be discerned by comparing survival data in tables 1 (overall analysis) and 2 (subgroup analysis). There is no mention of any plan to perform this subgroup analysis in the methods section - therefore there is no guarantee that this subgroup wasn't specifically formulated by data-dredging to elicit a presupposed conclusion. This is an excellent example of why unplanned subgroup analysis should not be accepted as a basis for scientific conclusions. The Editors of Chest accepted a letter we wrote in regards to this issue, so if you're interested, look for the authors reply in a subsequent issue of Chest.

Angus DC, van der Poll T. Severe sepsis and septic shock. N Engl J Med. 2013;369(9):840-51. [CrossRef] [PubMed]

Our thanks to Hargobind Khurana who helped us summarize this review article. For the sake of brevity, I will just relate a few comments that came forward in our discussion most of these relate to treatment. Table 2 of the article reviews current treatment guidelines from the Surviving Sepsis Campaign. Initial fluid resuscitation for patients with hypotension and suspected tissue hypoperfusion should rapidly deliver $\geq 30 \mathrm{~mL} / \mathrm{Kg}$ of crystalloid - and should continue so long as there is hemodynamic improvement. Of note, the definition of "hemodynamic improvement" is clearly moving beyond simply achieving a central venous pressure $>8 \mathrm{cmH}_{2} \mathrm{O}$ (a parameter that has been convincingly shown to not be predictive of fluid responsiveness). The level of evidence for this recommendation is only "C" - it is somewhat incredible that in 2013 , we still don't have better evidence to guide fluid resuscitation of septic shock. Of 35 recommendations listed in the table 2, only three are based on level "A" evidence: low tidal volume mechanical ventilation, use of ventilator weaning protocols, and use of a glucose management protocol. Nineteen of thirty-five (54\%) are level C or ungraded. The authors contend that our limited success in establishing high-level evidence for the management of sepsis might be explained by flaws in our current research strategy, which focuses on heterogeneous patient populations, with highly variable pathogens, clinical syndromes, and host immune responses. As more is learned about this 
heterogeneity, it might be found that treatment can be focused more specifically to the host-pathogen interaction - this approach may be more likely to succeed in clinical trials and at the bedside.

Dellinger RP, Townsend SR. Point: are the best patient outcomes achieved when ICU bundles are rigorously adhered to? Yes. Chest. 2013;144(2):372-4. [CrossRef] [PubMed]

Marik PE, Raghunathan K, Bloomstone J. Counterpoint: are the best patient outcomes achieved when ICU bundles are rigorously adhered to? No. Chest. 2013;144(2):374-8. [CrossRef] [PubMed]

Sandra Till did a good job summarizing this set of editorials. Dellinger and Marik both make good points - and probably most of our participants learned a few arguments for and against their personal stand on this issue. In very brief summary, Dellinger points out that bundles are not perfect, but are as evidenced-based as the current state of the literature permits. They promote consistency, help change practice behaviors, and provide compliance outcomes that are measurable. Marik points out that bundle elements frequently have poor quality evidence to support them, and that the concept of measuring compliance with the entire bundle as a measure of quality of care is flawed, because failure to comply with any single bundle element, even the most poorly supported, is interpreted as a failure to provide good care. Dr. O'Hea pointed out that chasing bundle compliance can sometimes distract us from what's most important for the individual patient.

Regardless which side of this issue you stand on, the use of bundle compliance as a measure of quality of care in the ICU is not likely to go away anytime soon. I think one of the most important things we can do is to express some local autonomy, and practice common sense in our definition of bundle compliance. For instance, it is highly unlikely that achieving a particular CVP is a specific measure of good ICU care - it is much more likely that good care entails paying careful attention to the patient's perfusion state and volume status in order to guide ongoing resuscitation, by whatever valid methods are employed. We might soon incorporate this concept by allowing use of inferior vena cava ultrasonography, and other appropriate methods, as a surrogate for CVP measurements, when determining bundle compliance. This will be a step in the right direction.

Page VJ, Ely EW, Gates S, et al. Effect of intravenous haloperidol on the duration of delirium and coma in critically ill patients (Hope-ICU): a randomised, doubleblind, placebo-controlled trial. Lancet Respir Med 2013;1: 515-23. [CrossRef]

This study was a double blind, randomized controlled trial that enrolled 142 general adult ICU patients to receive either haloperidol $2.5 \mathrm{mg}$ IV Q8 hourly or placebo, irrespective of delirium or coma state. Study treatment was continued until discharged from ICU, or awake for 2 days, for a maximum of 14 days. All patients also received fentanyl or propofol infusions titrated to Richmond agitation sedation scale target of 0 to -1 if needed. Patients in either group could also receive haloperidol doses as needed 
for agitation. The main outcome variable was the incidence of delirium as determined by the confusion assessment method (CAM-ICU). All but one patient was included in the intention to treat analysis. There was no difference in delirium free days, coma free days, mortality, or QT prolongation.

The study was well designed. Treatment groups did not differ in baseline characteristics. All but one patient was followed into the intension to treat analysis. Recruitment achieved a sample size with better than $80 \%$ power to demonstrate a clinically significant benefit. Other important outcomes including 21 potential drug side effects were assessed.

The conclusion of this study is not surprising. Haloperidol, and other sedation and antiagitation medications all have negative effects on cognitive function. It is much more likely that haloperidol and related medications act by simply reducing agitated behavior, making the patient more docile - rather than by clearing the mind. Prevention of delirium is most likely best achieved by avoiding or minimizing sedation medications, and other medications that have negative CNS effects, and by providing an environment in which the patient's orientation is supported. This might best be accomplished by good nursing-patient interaction, presence of family and friends, maintenance of normal sleep/wake cycles, proper use of analgesia, and other common sense measures.

Robert A Raschke MD MS Associate Editor 